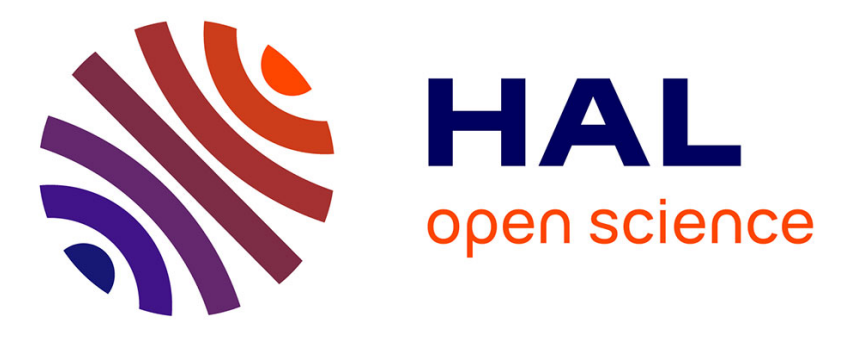

\title{
Doppler effect in the underwater acoustic ultra low frequency band
}

\author{
Abdel-Mehsen Ahmad, Michel Barbeau, Joaquin Garcia-Alfaro, Jamil Kassen, \\ Evangelos Kranakis, Steven Porretta
}

\section{- To cite this version:}

Abdel-Mehsen Ahmad, Michel Barbeau, Joaquin Garcia-Alfaro, Jamil Kassen, Evangelos Kranakis, et al.. Doppler effect in the underwater acoustic ultra low frequency band. ADHOCNETS 2017: 9th International Conference on Ad Hoc Networks, Sep 2017, Niagara Falls, Canada. pp.3 - 12, 10.1007/978-3-319-74439-1_1 . hal-01699512

\section{HAL Id: hal-01699512 \\ https://hal.science/hal-01699512}

Submitted on 2 Feb 2018

HAL is a multi-disciplinary open access archive for the deposit and dissemination of scientific research documents, whether they are published or not. The documents may come from teaching and research institutions in France or abroad, or from public or private research centers.
L'archive ouverte pluridisciplinaire HAL, est destinée au dépôt et à la diffusion de documents scientifiques de niveau recherche, publiés ou non, émanant des établissements d'enseignement et de recherche français ou étrangers, des laboratoires publics ou privés. 


\title{
Doppler Effect in the Underwater Acoustic Ultra Low Frequency Band
}

\author{
Abdel-Mehsen Ahmad ${ }^{1}$, Michel Barbeau ${ }^{2}$, Joaquin Garcia-Alfaro ${ }^{3}$, Jamil \\ Kassem $^{1}$, Evangelos Kranakis ${ }^{2}$, and Steven Porretta ${ }^{2}$ \\ 1 School of Engineering, Lebanese International University, Bekaa, Lebanon. \\ 2 School of Computer Science, Carleton University, Ottawa, ON, Canada, K1S 5B6. \\ 3 Telecom SudParis, CNRS Samovar, UMR 5157, Evry, France.
}

\begin{abstract}
We address communications between Autonomous Underwater Vehicles (AUVs), Underwater Sensors (USs) and remote operators. We assume the use of acoustic waves. Due to the Doppler effect, the communication frequency depends on the relative motion between the participants. We are interested in the Ultra Low Frequency (ULF) range, from 0.3 to $3 \mathrm{kHz}$. We relate the Doppler effect to the half-power bandwidth, versus distance. Numeric simulations are conducted. We show that the Doppler shift is significant with respect to the half-power bandwidth in the ULF band, for long distance communications.
\end{abstract}

\section{Introduction}

Autonomous Underwater Vehicles (AUVs) and Underwater Sensors (USs) use acoustic waves to communicate. We are interested in the Ultra Low Frequency (ULF) range, 0.3 to $3 \mathrm{kHz}(\mathrm{kHz})$, underwater communications. The ULF band is interesting because the attenuation is lower, relative to higher frequencies. Hence, there is more potential for long range communications. For instance, Freitag et al. [1] have been able to make contact at a distance of $400 \mathrm{~km}$ at $900 \mathrm{~Hz}$. On the other hand, the half-power bandwidth is narrow in the ULF band. As a consequence, solely extremely low rate data streams can be supported. Another communication impairment is the Doppler effect. It is created by relative motions between acoustic sources and receivers. Given the narrow half-power bandwidth and slow propagation speed of underwater acoustic waves, one may expect a significant Doppler effect in the ULF band. The goal of this work is to characterize the importance of the Doppler effect in various underwater communication scenarios in the ULF band. Some questions addressed are: What is the maximum Doppler shift that can be expected on underwater links in the ULF band? What is the maximum frequency drift that can happen during the reception of a data frame? Through a number of scenarios, we show that the Doppler shift is significant in the ULF band for long distances, relative to the narrow half-power bandwidth.

Section 2 provides background on ULF underwater acoustic communications. Section 3 discusses the Doppler effect. Section 4 presents our experimental scenarios and results. Section 5 concludes. 


\section{ULF Underwater Acoustic Communications}

Attenuation is an important underwater acoustic communication impairment. The main causes are conversion of acoustic energy into heat and geometrical spreading. The magnitude of attenuation is represented in the Thorp's model [24]. For long distance underwater communications, the ULF band is preferable because there is less attenuation at the lower end of the acoustic spectrum.

Figure 1(a) plots the attenuation as a function of distance for selected frequencies in the ULF band. Realistically, for long range underwater acoustic communications, solely the use of low frequencies can be envisioned. For instance, Freitag et al. [1] have been able to achieve communication over a $400 \mathrm{~km}$ range at $900 \mathrm{~Hz}$.

Another important fact is the gradient of the attenuation versus frequency. The transmission loss rapidly increases for higher frequencies. It limits the operating bandwidth. This constraint is captured by the concept of half-power bandwidth. The half-power bandwidth is commonly used to define cutoff frequencies and bandwidths of filters by using frequency response curves, using $3 \mathrm{~dB}$ points in the frequency response of a band-pass filter [5].

Figure 1(b) shows the half-power bandwidth for selected ULF frequencies versus distance. Firstly, the relationship between frequency, half-power bandwidth and distance is not linear. Secondly, at very long ranges (e.g., $400 \mathrm{~km}$ ), the half-power bandwidth is very narrow, i.e., around $100 \mathrm{~Hz}$.

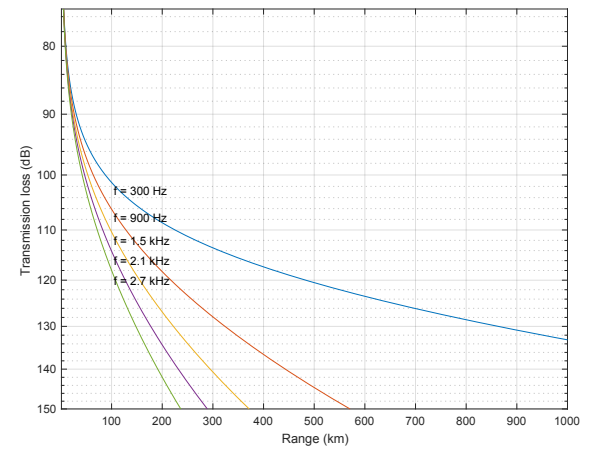

(a) Attenuation.

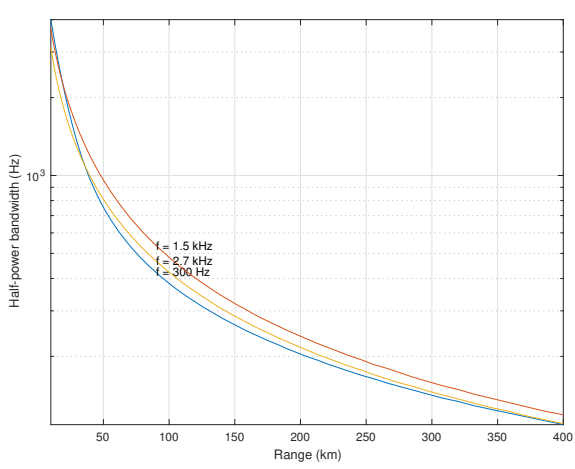

(b) Half-power bandwidth.

Fig. 1. (a) Attenuation and (b) half-power bandwidth for selected frequencies in the underwater acoustic ULF band. We can observe the relation between the half-power bandwidth and the frequency, with respect to the range. For a range less than $17 \mathrm{~km}$, the half-power bandwidth is better for low frequencies $(300 \mathrm{~Hz})$ than for higher frequencies with the same range. 


\section{Doppler Effect}

The Doppler effect shifts the frequency, from the receiver point of view, because of a transmitter-receiver delay change during data transmission. This happens because either the transmitter or the receiver are mobile. Their relative separation distance is not constant. Let $v(\mathrm{~m} / \mathrm{s})$ be the relative velocity between a transmitter and a receiver. It is positive when they are getting closer, negative when moving away. Let $c$ be the signal propagation speed $(\mathrm{m} / \mathrm{s})$. At nominal frequency $f_{0} \mathrm{~Hz}$, the variation of frequency due to the Doppler effect is [6]:

$$
\delta f=f_{0} \frac{v}{c} \mathrm{~Hz}
$$

Figure 2 depicts the maximum Doppler shift for selected frequencies in the ULF range. We assume that the relative speed varies from zero to eight knots. This range is consistent with the values reported by Robert et al. [7] about

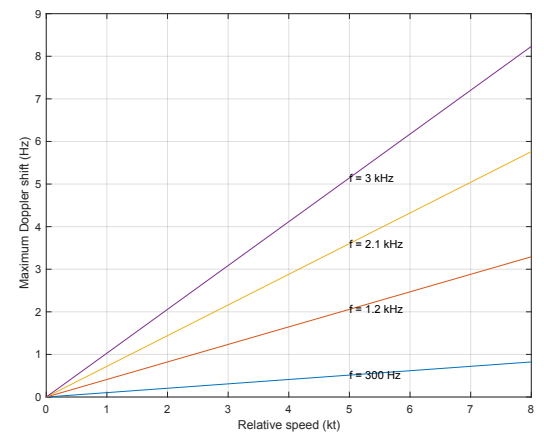

(a) Colateral motions.

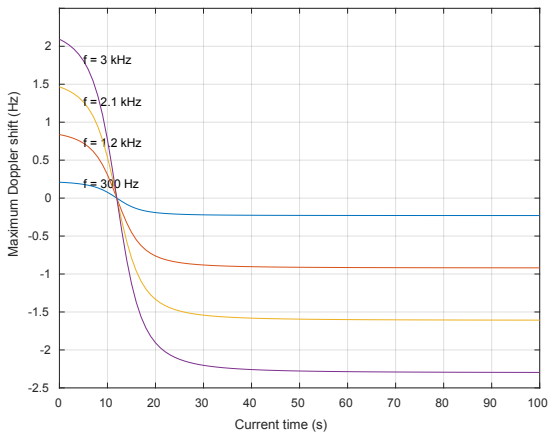

(b) Transverse motions.

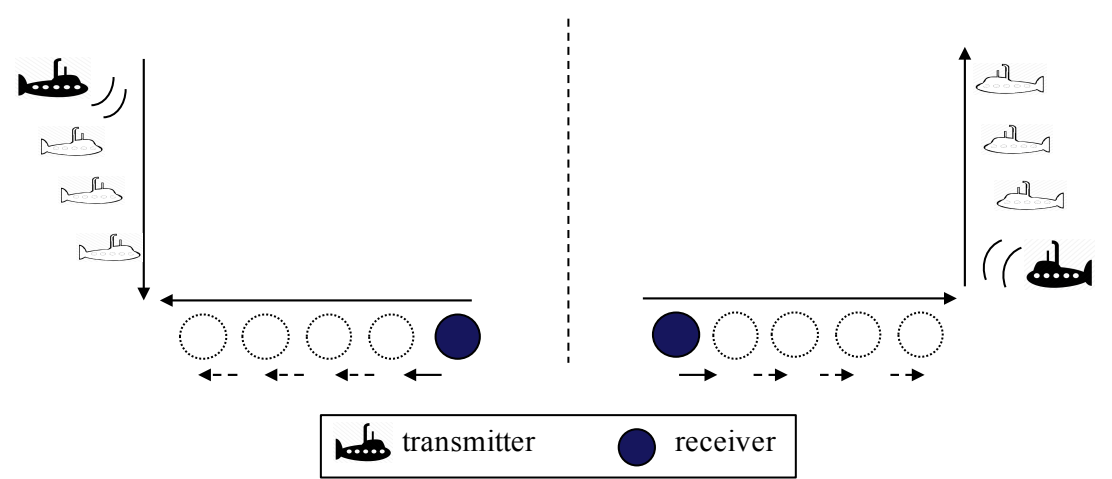

(c) Scenario for the transverse motion of a transmitter and a receiver.

Fig. 2. Maximum Doppler shift in the ULF range, assuming mobile transmitter and receiver moving either on the same axis (a) or in transverse directions (b). The scenario for the transverse movement between the transmitter and receiver is depicted in (c). 
speed range of AUVs. Figure 2(a) shows that the Doppler shift turns out to be linear with respect to the relative speed, if we assume that both transmitter and receiver are moving along the same axis. Figure 2(b) makes the assumption that the transmitter and receiver move in transverse directions (i.e., transmitter and receiver move in opposite directions with respect to a reference axis) with constant speeds and as in the scenario shown in Figure 2(c). After nine seconds, both of them arrive at the same point, where the Doppler shift becomes null. Then, they move away from each other, causing an increase in the Doppler shift.

\section{Experimental Scenarios and Results}

Experiments are conducted using numeric simulations via BELLHOP [8,9]. We assume situations in which one mobile transmitter (representing an AUV) is continuously transmitting acoustic waves at a specific frequency. At an initial distance, the waves are processed by an array of receivers (underwater sensors equipped with acoustic hydrophones). The array of receivers also move with respect to a mobility model (e.g., a sinusoidal movement). At each instance, one or more receivers process a series of multipath components that are summed up together at the receiver side. These multipath components consist of a straight line-of-sight (LOS) ray and multiple reflected and refracted rays. The rays are generated using BELLHOP. Each ray comes with different delay shifts, causing different frequency shifts. Our goal is to study variations of Doppler shifts and estimate the detection accuracy in a series of communication scenarios. The source code of the simulations is available online at http://j.mp/UWtmpgit. Next, we present our main scenarios.

\subsection{Doppler Shift for a Transmitter-receiver Pair}

Figure 3(a) depicts our first scenario. It consists of two underwater devices: one acting as a transmitter $(T)$ and the other acting as a receiver $(R) . R$ moves according to a sinusoidal model along the $z$ axis ( $\operatorname{such}$ that $z=A \sin \left(\frac{t \cdot \pi}{60}\right)$ ). The trajectory of $T$ is based on the Caruso et al. model [10,11], which simulates a movement with one degree of freedom. The Doppler shift is derived according to Equation (1) ( $c$ is $1500 \mathrm{~m} / \mathrm{s}$ ). The distance between the two devices is derived by substituting their three-dimensional coordinates in the equation $\sqrt{\left(x_{2}-x_{1}\right)^{2}+\left(y_{2}-y_{1}\right)^{2}+\left(z_{2}-z_{1}\right)^{2}}$. Change of distances are used to compute the relative velocity between $T$ and $R$. The data is produced at one sample per second.

Figure 4 plots the Doppler shifts for times $t=0$ to $t=50$ seconds. Figure 4(a), the amplitude $A$ of $R$ is $10 \mathrm{~m}$. The figure shows a slight frequency

shift, that is one $\mathrm{Hz}$ at its maximum. In Figure 4(b)), the amplitude $A$ of $R$ is $50 \mathrm{~m}$. The Doppler shift becomes noticeable, as it peaks at $35 \mathrm{~Hz}$.

Scenario 1.1 - Mobile Transmitter, Stationary Receiver. In Figure 5(a), the transmitter is assumed to follow a sinusoidal motion with frequency $f_{m}=$ 
Sea surface

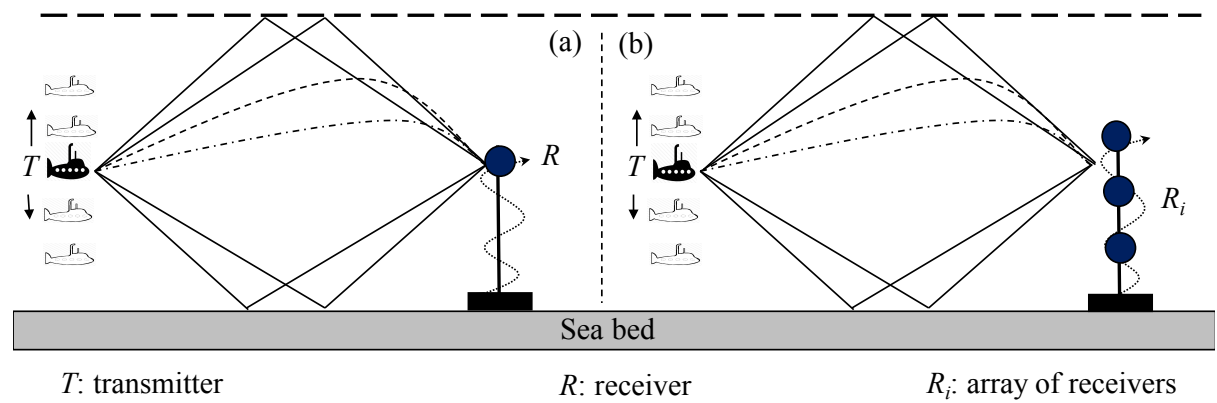

Fig. 3. Experimental scenarios. (a) Communication between one transmitter and one receiver. (b) Communication between one transmitter and one array of receivers. The receivers moves according to a sinusoidal model along the $z$ axis. The transmitter moves according to the model in $[10,11]$, which simulates a one-degree freedom of movement. Straight lines represent either reflected or refracted rays. Dashed lines represent eigenrays, i.e., no reflections nor refractions. Some videocaptures of the assumed mobility patterns are available at: http://j.mp/UWtransmitters and http://j.mp/UWreceivers.

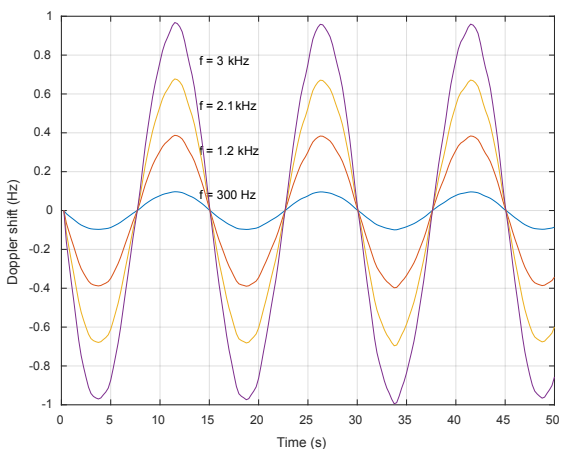

(a) $R$ 's motion amplitude $A$ is $10 \mathrm{~m}$.

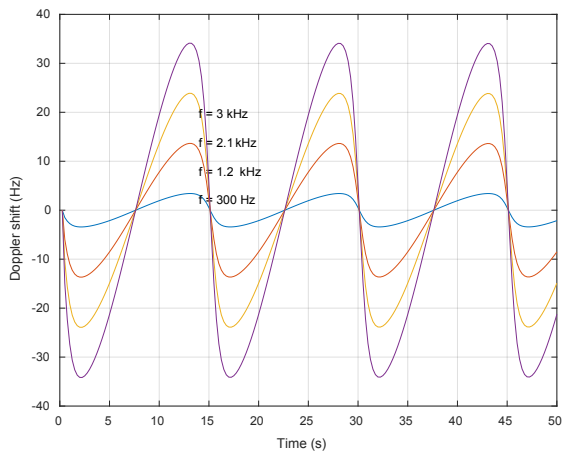

(b) $R$ 's motion amplitude $A$ is $50 \mathrm{~m}$.

Fig. 4. Doppler shift at different receiver positions for selected ULF frequencies. The receiver moves according to a sinusoidal law. 
$0.1 \mathrm{~Hz}$ and amplitude $A$ of $10 \mathrm{~m}$. The receiver is stationary. The transmitter is moving along a line with an invariant angle $\beta$ relative to the $x$-axis. This line makes a variable angle $\alpha$ with a transmitter-receiver line. Since the transmitter is moving along an angle $\beta$ with respect to the $x$-axis, we can compute the horizontal position $x$ of the transmitter as follows:

$$
x=\cos \beta \cdot A \cdot \cos \left(\frac{\pi t}{f_{m}}\right) \mathrm{m}
$$

and its depth $d$ as:

$$
d=\sin \beta \cdot A \cdot \cos \left(\frac{\pi t}{f_{m}}\right) \mathrm{m}
$$

The frequency at the receiver $f_{r}$ is computed using the following formula:

$$
f_{r}=\frac{c}{c-v_{s} \cos (\alpha)} \cdot f_{s} \mathrm{~Hz}
$$

where $f_{s}$ is the frequency of the transmitter, $c$ is the signal propagation speed (assumed to be $1500 \mathrm{~m} / \mathrm{s}$ ), and $v_{s}$ is the velocity of the transmitter. The value of $v_{s}$ is obtained by computing $\frac{\delta d}{\delta t}$, where $\delta d$ is the change of distance during an interval of time $t$.

Scenario 1.2 - Mobile Receiver, Stationary Transmitter. In Figure 5(b), the receiver is moving with velocity $v_{r}$ along a line with constant angle $\beta$, relative to the $x$-axis. Variable $\alpha$ is the angle between this line and transmitter-receiver line. The receiver is assumed to follow a sinusoidal motion with frequency 0.1 $\mathrm{Hz}$ and amplitude of $10 \mathrm{~m}$. The transmitter is stationary. Same operations as in Scenario 1.1 are computed, except for deriving the frequency at the receiver $f_{r}$, which is computed using the following equation:

$$
f_{r}=\left(1+\frac{v_{r} \cdot \cos \alpha}{c}\right) \cdot f_{s}
$$

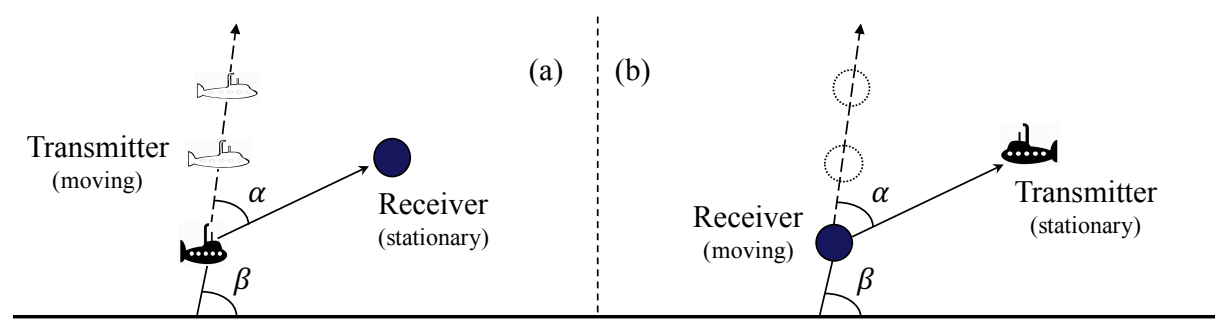

Fig. 5. (a) Scenario 1.1, moving transmitter and stationary receiver. (b) Scenario 1.2, moving receiver and stationary transmitter. 


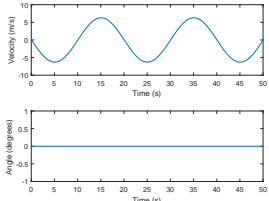

(a) $\beta=0^{\circ}$
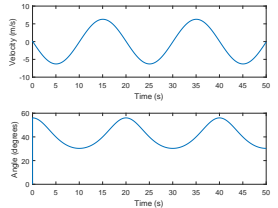

(b) $\beta=40^{\circ}$
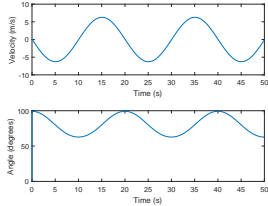

(c) $\beta=80^{\circ}$
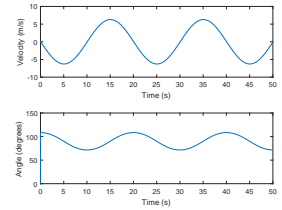

(d) $\beta=90^{\circ}$

Fig. 6. Mobility characteristics for the Doppler shift experiments assuming $1.5 \mathrm{kHz}$ for the frequency; and 0 to $90^{\circ}$ as angle $\beta$.

Figures 6 and 7 show the simulation results. Figure 6 shows the variation of both angle and velocity of movement. The moving receiver and moving transmitter scenarios produced the same results. Figure 7 shows the variation of the Doppler shift for four $\beta$ angles: $\left(0^{\circ}, 40^{\circ}, 80^{\circ}\right.$ and $\left.90^{\circ}\right)$. In Figure $7(\mathrm{f})$ velocity is increased $40 \times$.

\subsection{Doppler Shifts and Attenuation Between one Transmitter and Several Receivers}

We study the Doppler shift assuming the existence of multiple receivers, as depicted in Figure 3(b). Different scenarios are discussed.

Assuming that the transmitter is positioned at $15 \mathrm{~m}$ deep, five receivers are placed $30 \mathrm{~m}$ away from the transmitter, at depths zero, 10, 20, 30, and $40 \mathrm{~m}$. The array of receivers is stationary. The following cases are considered:

1. The transmitter is moving along a line parallel to the $x$-axis, where $x=$ $10 \cos (0.1 \pi t) \mathrm{m}$. Depth is constant at $15 \mathrm{~m}$.

2. The transmitter is moving along a line parallel to the vertical axis, where depth $d=15+10 \cos (0.1 \pi t) \mathrm{m}$ and $x=0 \mathrm{~m}$.

Let $v$ be the velocity of the transmitter. The following equation is used to compute the Doppler shift:

$$
\delta f=\frac{c}{c-v \cos \alpha}
$$

where $\alpha$ is the angle between the line along which the transmitter is moving and transmitter to receiver line.

Figure 8 shows the results of the simulations and the velocity and mobility patterns assumed during the experiments. When angle $\beta$ is set to $90^{\circ}$, receivers are far from the transmitter, i.e., at distance greater than the amplitude of the sinusoidal motion of the transmitter, each receiver is either above or below the transmitter. If the transmitter is moving up, then it gets closer to the receiver placed at the higher depth. It moves away from the receivers placed at the bottom of the array. It is reflected in the Doppler shift. When the motion of the transmitter is along the $x$-axis, all the receivers experience similar delays. At depths 10 to $20 \mathrm{~m}$, the Doppler shift is the same as at depths 0 and $30 \mathrm{~m}$ since the transmitter is moving in the middle at depth $15 \mathrm{~m}$. 


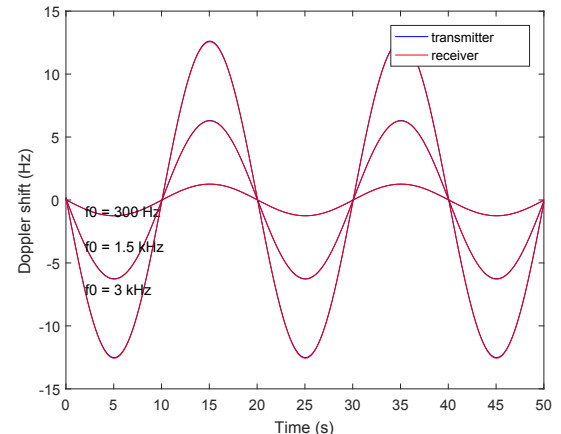

(a) $\beta=0^{\circ}$

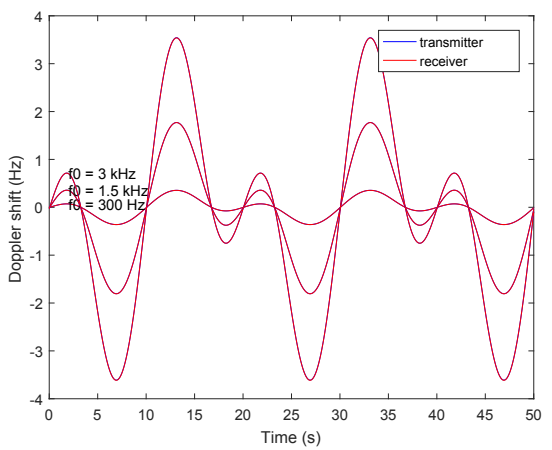

(c) $\beta=80^{\circ}$

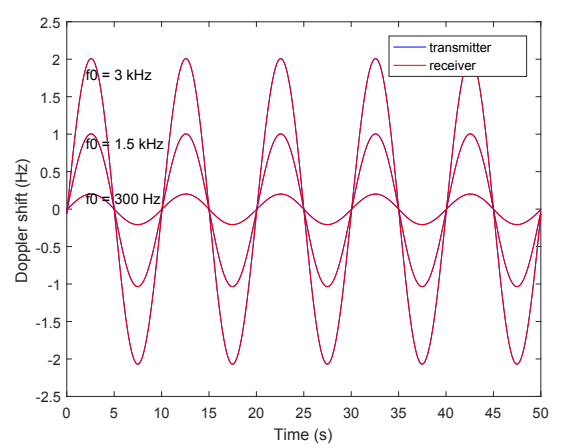

(e) Normal velocity

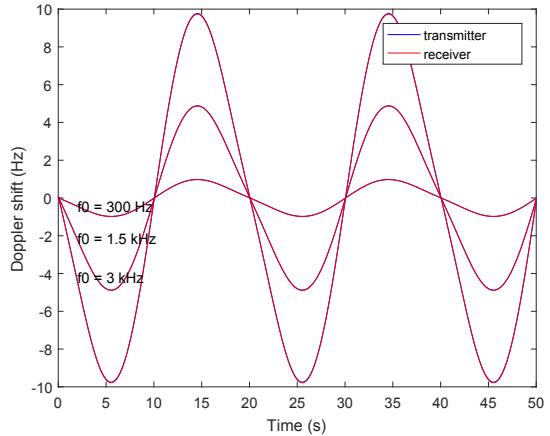

(b) $\beta=40^{\circ}$

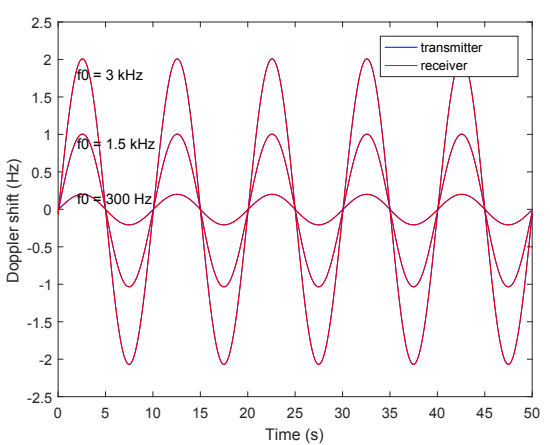

(d) $\beta=90^{\circ}$

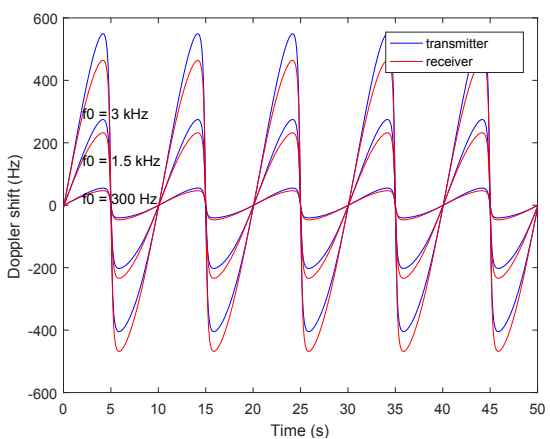

(f) 40x velocity increase

Fig. 7. Doppler shift experiments at $1.5 \mathrm{kHz}$ and angles $0^{\circ}$ to $90^{\circ}$. 


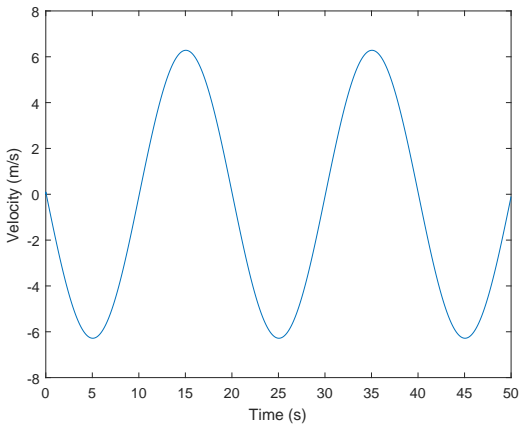

(a) Velocity of the transmitter.

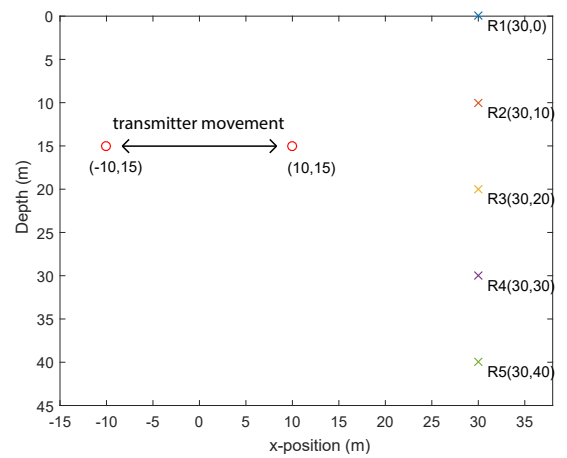

(c) $\beta=0^{\circ}, x=10 \sin \left(\frac{\pi t}{10}\right)$.

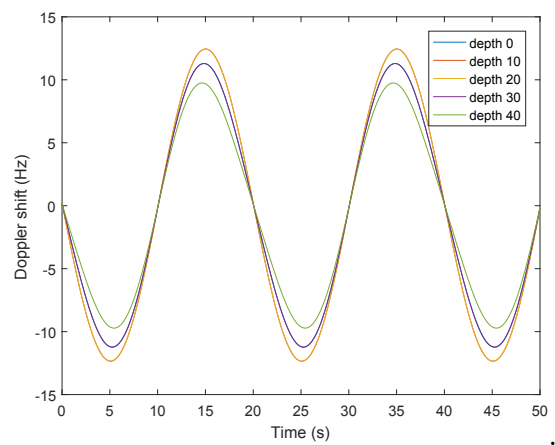

(e) Shifts, $\beta=0^{\circ}, x=10 \sin \left(\frac{\pi t}{10}\right)$.

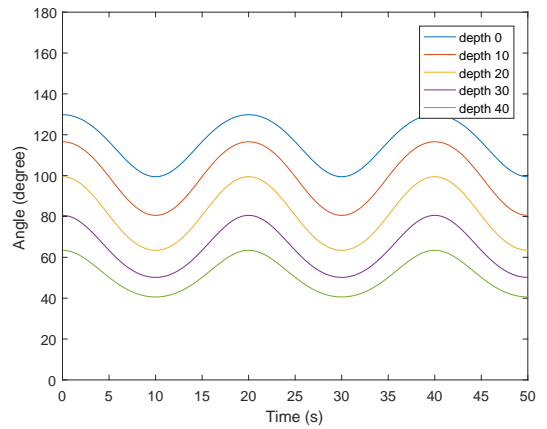

(b) Mobility pattern of the transmitter.

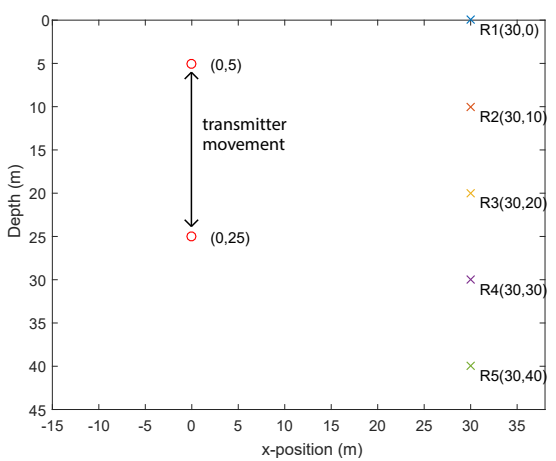

(d) $\beta=90^{\circ}, d=15+10 \sin \left(\frac{\pi t}{10}\right)$.

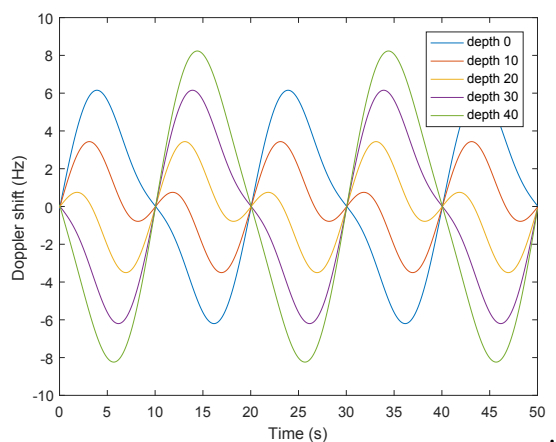

(f) Shifts, $\beta=90^{\circ}, d=15+10 \sin \left(\frac{\pi t}{10}\right)$.

Fig. 8. Doppler shifts affecting transmitters (a,b) moving along either the $x$-axis (c),(e) or $y$-axis (d),(f). 


\section{Conclusion}

We have addressed acoustic communications between AUVs, USs and remote operators. We studied scenarios comprising one transmitter and one or several receivers. Due to the mobility of nodes, the Doppler effect changes the communication frequency. We focused on the ULF band, i.e., the frequency range 0.3 to $3 \mathrm{kHz}$. Numeric simulations confirm the importance of the Doppler shift. We have a maximum Doppler shift of $10 \mathrm{~Hz}$ in the scenarios we studied. It is negligible for short and medium ranges. It is, however, significant with respect to the half-power bandwidth for long distance communications (400 km). It corresponds to $10 \%$ of the half-power bandwidth. Since attenuation also depends on frequency [2-4], a positive Doppler shift increases the frequency and augments the attenuation, and vice-versa. In our simulations, the Doppler effect on the

attenuation bandwidth is not significant. The source code of the simulations is available online at http://j.mp/UWtmpgit.

\section{References}

1. L. Freitag, J. Partan, P. Koski, and S. Singh. Long range acoustic communications and navigation in the arctic. In OCEANS 2015 - MTS/IEEE Washington, pages 1-5, October 2015.

2. W.H. Thorp. Analytic description of the low frequency attenuation coefficient. The Journal of the Acoustical Society of America, 42:270, 1967.

3. W.H. Thorp. Deep ocean sound attenuation in the sub and low kilocycle per second region. The Journal of the Acoustical Society of America, 38(4):648-654, 1965.

4. W.H. Thorp and D.G. Browning. Attenuation of low frequency sound in the ocean. Journal of Sound and Vibration, 26:576-578, February 1973.

5. Baisheng $\mathrm{Wu}$. A correction of the half-power bandwidth method for estimating damping. Archive of Applied Mechanics, 85(2):315-320, 2015.

6. Xavier Lurton. An Introduction to Underwater Acoustics: Principles and Applications. Springer, 2002.

7. Robert W. Button, John Kamp, Thomas B. Curtin, and James Dryden. A survey of missions for unmanned undersea vehicles, 2009.

8. Michael B. Porter. The BELLHOP manual and user's guide. http://oalib. hlsresearch.com/Rays/index.html, 2011.

9. Orlando Camargo Rodriguez. General description of the BELLHOP ray tracing program. http://oalib.hlsresearch.com/Rays/index.html, 2008.

10. Antonio Caruso, Francesco Paparella, Luiz Filipe M Vieira, Melike Erol, and Mario Gerla. The meandering current mobility model and its impact on underwater mobile sensor networks. In 27th Conference on Computer Communications (INFOCOM 2008), pages 221-225. IEEE, 2008.

11. Antonio Caruso. Simple jet meandering model library. https://github.com/ antoniocaruso/smm, 2014. 